\title{
Accuracy of the spin sum rule in XMCD for the transition-metal $L$ edges from manganese to copper
}

\author{
Cinthia Piamonteze \\ Swiss Light Source, Paul Scherrer Institut, CH-5232 Villigen PSI, Switzerland
}

Piter Miedema and Frank M. F. de Groot

Inorganic Chemistry and Catalysis, Utrecht University, Sorbonnelaan 16, 3584 CA Utrecht, The Netherlands (Received 6 August 2009; revised manuscript received 28 September 2009; published 10 November 2009)

\begin{abstract}
The effective spin sum rule is widely used in the quantitative analysis of $\mathrm{x}$-ray magnetic circular dichroism spectra. Here, this important, though imperfect, sum rule is reviewed with a detailed analysis of the various sources for errors and deviations. The simulations confirm that the final state effects of the core level spin-orbit coupling and the core-valence exchange interactions (multiplet effects) are linearly related with the effective spin sum-rule error. Within the charge transfer multiplet approach, we have analyzed these effects, in combination with the interactions affecting the magnetic ground state, including the crystal field strength, the charge transfer effects, the exchange (magnetic) field, and the $3 d$ spin-orbit coupling. We find that for the late transition-metal systems, the error in the effective spin moment is between $5 \%$ and $10 \%$, implying that for covalent and/or metallic systems the effective spin sum rule is precise to within $5-10 \%$. The error for $3 d^{5}$ systems is $\sim 30 \%$ and for $3 d^{4}$ systems, the error is very large, implying that, without further information, the derived effective spin sum-rule values for $3 d^{4}$ systems have no meaning.
\end{abstract}

DOI: $10.1103 /$ PhysRevB.80.184410

PACS number(s): 74.25.Ha, 11.55.Hx, 72.80.Ga, 78.70.Dm

\section{INTRODUCTION}

The X-ray magnetic circular dichroism (XMCD) sum rules have been introduced by Thole et al. in 1992 (Ref. 1) and Carra et al. in $1993 .^{2}$ Thole et al. showed that the integral over the XMCD signal of a given edge allows for the determination of the ground state expectation values of the orbital moment $\left\langle L_{z}\right\rangle$ and Carra et al. introduced a second sum rule for the effective spin moment $\left\langle S E_{z}\right\rangle$. The sum rules apply to a transition between two well-defined shells, for example, the transition from a $2 p$ core state to $3 d$ valence states in transition-metal systems. These $3 d$ valence states are assumed to be separable from other final states, for example, the $4 s$ conduction band states that can be reached via a $2 p 4 s$ transition. This implies that the $2 p 3 d$ edge absorption must be separated from the $2 p 4 s$ and other $2 p$-continuum transitions. In general, it is assumed that continuum transitions can be described as an edge step followed by a constant cross section.

The XMCD sum rules have been reviewed in a number of publications. ${ }^{3-6}$ Here we briefly introduce the main aspects. The integrated $2 p 3 d$ x-ray absorption spectrum is proportional to the number of empty $3 d$ states $\left(\left\langle n_{h}\right\rangle\right)$,

$$
\begin{array}{r}
\int \mu \equiv \int\left(\mu_{+1}+\mu_{0}+\mu_{-1}\right)=\frac{C}{5}\left\langle N_{h}\right\rangle \\
\text { with } \int \mu=\int_{L_{3}+L_{2}} \mu(\Omega) d \Omega .
\end{array}
$$

The absorption cross section $(\mu)$ is integrated over a certain energy range $(\Omega)$ that covers the complete $L_{2,3}$ edge. $C$ is a constant factor including the radial matrix element of the dipole transition. The integrated circular dichroism spectrum is defined as the absorption of left circular polarized, positive helicity, $\mathrm{x}$ rays $\left(\mu_{+1}\right)$ minus the absorption of right circular polarized, negative helicity, $\mathrm{x}$ rays $\left(\mu_{-1}\right)$. In case of a $2 p 3 d$ transition this yields

$$
\int_{L_{3}+L_{2}}\left(\mu_{+1}-\mu_{-1}\right)=-\frac{C}{10}\left\langle L_{z}\right\rangle .
$$

This XMCD sum rule implies that one can directly determine the orbital moment from the difference of positive $\left(\mu_{+1}\right)$ and negative $\left(\mu_{-1}\right)$ helicity $\mathrm{x}$ rays. Because in most soft $\mathrm{x}$-ray experiments one uses yield detection schemes, the absolute absorption cross section is not measured and only a relative signal is measured. A solution is to normalize the XMCD signal by the absorption edge. This defines the orbital moment sum rule as

$$
\left\langle L_{z}\right\rangle=-\frac{\int_{L_{3}+L_{2}}\left(\mu_{+1}-\mu_{-1}\right)}{\int \mu} 2\left\langle N_{h}\right\rangle .
$$

It is important if one could also determine the spin moment and this is indeed possible with an additional sum rule. However, this effective spin sum rule has some additional complications as is discussed below,

$$
\left\langle S E_{z}\right\rangle=\frac{\int_{L_{3}}\left(\mu_{+1}-\mu_{-1}\right)-2 \int_{L_{2}}\left(\mu_{+1}-\mu_{-1}\right)}{\int \mu} \frac{3}{2}\left\langle N_{h}\right\rangle .
$$

The effective spin moment $\left\langle S E_{z}\right\rangle$ is given as

$$
\left\langle S E_{z}\right\rangle=\left\langle S_{z}\right\rangle+\frac{7}{2}\left\langle T_{z}\right\rangle,
$$

where $\left\langle T_{z}\right\rangle$ is the spin-quadrupole coupling. If this sum rule is used to determine the spin moment $\left\langle S_{z}\right\rangle$ one has to assume that $\left\langle T_{z}\right\rangle$ is zero or $\left\langle T_{z}\right\rangle$ must be known from other experiments or theoretically approximated. The effective spin sum rule makes an additional approximation that the $L_{3}$ and the $L_{2}$ edges are not mixed and well separated. The edges must be well separated in energy because otherwise there is no 
clear method to divide the spectrum into $L_{3}$ and $L_{2}$. Moreover, the two edges must be pure $2 p_{3 / 2}$ and $2 p_{1 / 2}$. Throughout this paper we will discuss two different sum-rule errors:

(a) the error in the spin moment $\left\langle S_{z}\right\rangle$ and

(b) the error in the effective spin moment $\left\langle S E_{z}\right\rangle$.

The error in the effective spin moment $\left\langle S E_{z}\right\rangle$ is, as will be shown below, caused by the mixing of the $L_{3}$ and $L_{2}$ edges. The error in the spin moment $\left\langle S_{z}\right\rangle$ has, in addition, the effect of $7 / 2\left\langle T_{z}\right\rangle$.

Since the derivation of the effective spin sum rule, its accuracy and validity have been discussed. The effective spin sum rule has been theoretically simulated and tested by Teramura et $a l .{ }^{7}$ They calculated the expectation values of the effective spin $\left\langle S E_{z}\right\rangle$ and compared them with simulated effective spin sum-rule values $\left[S E_{z}^{\text {sum }}\right]$. van der Laan et al. ${ }^{8}$ used the ratio of the $G_{1}(p d)$ Slater integral and the core hole spin-orbit coupling to estimate the purity of the $L_{2}$ and $L_{3}$ edges and as such the accuracy of the effective spin-orbit sum rule. They found the largest error for the $L$ edges of $3 d$ transition metals. Also the $M_{4,5}$ edge of the rare earth has large errors, but the edges of the $4 d, 5 d$, and $5 f$ systems have negligible errors due to the mixing of the spin-orbit split components. ${ }^{8}$

Crocombette et al. ${ }^{9}$ also tested the effective spin sum rule theoretically. For an octahedral system at $300 \mathrm{~K}$ they found that the sum-rule value for $\left\langle S_{z}\right\rangle$ is $\sim 10 \%$ too small for $3 d^{6}$, $3 d^{7}$, and $3 d^{8}$. The errors increase to $28 \%$ and $56 \%$ too small for $3 d^{5}$ and $3 d^{4}$. In this paper the focus is on the role of the $\left\langle T_{z}\right\rangle$ operator and it was found that in octahedral symmetry, the value of $\left\langle T_{z}\right\rangle$ is determined by the $3 d$ spin-orbit coupling. Because the spin-orbit coupling is small, the value of $\left\langle T_{z}\right\rangle$ is close to zero at room temperature. $\left\langle T_{z}\right\rangle$ reaches larger values at temperatures where the $3 d$ spin-orbit coupling causes an uneven distribution over the states. At lower symmetry the value of $\left\langle T_{z}\right\rangle$ is essentially given by the occupation of the respective $3 d$ orbitals and it is essentially unaffected by the $3 d$ spin-orbit coupling. ${ }^{9}$ van der Laan et al. ${ }^{10}$ also discussed the role of $\left\langle T_{z}\right\rangle$ and its large value for small crystal field values. Wu et al. ${ }^{11,12}$ calculated the value of $\left\langle T_{z}\right\rangle$ for both the bulk and the surface of $3 d$ transition metals using density functional theory based band structure calculations. They found large values of $\left\langle T_{z}\right\rangle$ at the surface, yielding $\left\langle S_{z}\right\rangle$ errors up to $50 \%$ for the $\mathrm{Ni}(001)$ surface, solely due to the value of $\left\langle T_{z}\right\rangle$. Within this approximation, the error in $\left\langle S E_{z}\right\rangle$ is found to be small.

Goering et al. ${ }^{13}$ developed an element specific renormalization technique to derive the spin moment from the effective spin sum rule. The technique uses moment analysis to disentangle the $L_{3}$ and $L_{2}$ parts of the spectrum, yielding a correction factor for the spin moment. The various features of the $L_{2}$ and the $L_{3}$ edges are fitted simultaneously, which result in a deconvolution of the XMCD spectra into different excitation channels, interpreted by variations of the unoccupied density of states. Effectively, it is assumed that the deviation of the branching ratio from its statistical value of $2 / 3$ gives rise to a correction factor. In the discussion we analyze this assumption with respect to the calculated curves and their potential to derive a correction factor.

\section{METHOD}

\section{A. Ligand field multiplet calculations}

In case of the $3 d$ metal $L_{2,3}$ edges, the agreement between one-electron codes and the x-ray absorption spectral shape is, in general, poor. The reason for this discrepancy is that one does not observe the density of states in such X-ray absorption processes due to the strong overlap of the core wave function with the valence wave functions. In the final state of an x-ray absorption process one finds a partly filled core state, for example, a $2 p^{5}$ configuration. In case one studies a system with a partly filled $3 d$ band, for example, a $3 d^{8}$ system, the final state will have an incompletely filled $3 d$ band, which after the $2 p 3 d$ transition can be approximated as a $3 d^{9}$ configuration. The $2 p$ hole and the $3 d$ hole have radial wave functions that overlap significantly. This wave function overlap is an atomic effect that can be very large. It creates final states that are found after the vector coupling of the $2 p$ and $3 d$ wave functions. This effect is well known in atomic physics and actually plays a crucial role in the calculation of atomic spectra. Experimentally it has been shown that while the direct core hole potential is largely screened, these socalled multiplet effects are hardly screened in the solid state. This implies that the atomic multiplet effects are of the same order of magnitude in atoms and in solids. Ligand field theory is a model that is based on a combination of these atomic effects and the role of the surrounding ligand approximated with an effective electric field. The starting point of the crystal field model is to approximate the transition metal as an isolated atom surrounded by a distribution of charges that should mimic the system, molecule or solid, around the transition metal. ${ }^{14,15}$

\section{B. Charge transfer multiplet calculations}

Charge transfer effects are the effects of charge fluctuations in the initial and final states. The ligand field multiplet model uses a single $3 d^{N}$ configuration to describe the ground state and final state. One can combine this configuration with other low-lying configurations similar to the way configuration interaction works with a combination of Hartree-Fock matrices. In oxides and metals, a $3 d^{N}$ ground state is typically combined with a $3 d^{N+1} \underline{\varepsilon}$ configuration, where $\underline{\varepsilon}$ is a missing electron (hole) in a delocalized band or in a ligand state $(\underline{L})$.

\section{Procedure to determine the theoretical sum-rule values}

Within the ligand field multiplet (LFM) calculations, the transition-metal ion is defined with one configuration, $3 d^{N}$. The ground state expectation values of $\left\langle L_{z}\right\rangle,\left\langle S_{z}\right\rangle$, and $\left\langle T_{z}\right\rangle$ are calculated. These ground state expectation values are affected by the $3 d 3 d$ Slater integrals, the $3 d$ spin-orbit coupling, and the ligand field splitting.

The $2 p$ X-ray absorption and XMCD spectra are calculated. The spectral shape is, in addition to the ground state interactions mentioned above, determined by the $2 p$ core hole spin-orbit coupling and the $2 p 3 d$ Slater integrals. The orbital sum rule and the effective spin sum rules are applied 
to the calculated spectra. This theoretical sum-rule calculation uses the following assumptions:

(i) The division of the spectrum into its $L_{3}$ and $L_{2}$ components, similar as one would use for an experimental spectrum.

(ii) The addition of the calculated, unbroadened, stick values for both the $L_{3}$ and the $L_{2}$ edges.

(iii) The application of the effective spin sum rule [Eq. (4)]. This yields the theoretical sum-rule-derived value for $\left\langle S E_{z}\right\rangle$, defined as $\left[S E_{z}^{\text {sum }}\right]$. The theoretical sum-rule-derived value for the orbital moment is defined as $\left[L_{z}^{\text {sum }}\right]$.

(iv) The sum-rule values are compared with the calculated ground state values to determine the ratio $\left[S E_{z}^{\text {sum }}\right] /\left\langle S E_{z}\right\rangle$ and $\left[L_{z}^{\text {sum }}\right] /\left\langle L_{z}\right\rangle$.

(v) The value of $\left[L_{z}^{\text {sum }}\right] /\left\langle L_{z}\right\rangle$ is equal to 1 for all calculations performed, confirming the theoretical validity of the orbital moment sum rule.

\section{RESULTS}

The effective spin moment sum rule has been tested theoretically for $\mathrm{Mn}^{3+} 3 d^{4}, \quad \mathrm{Fe}^{3+} 3 d^{5}, \quad \mathrm{Fe}^{2+} 3 d^{6}, \quad \mathrm{Co}^{2+} 3 d^{7}$, $\mathrm{Ni}^{2+} 3 d^{8}$, and $\mathrm{Cu}^{2+} 3 d^{9}$. The procedure we use calculates for a given ground state their spin $\left\langle S_{z}\right\rangle$, orbital $\left\langle L_{z}\right\rangle$, and spinquadrupole $\left\langle T_{z}\right\rangle$ expectation values and compares them with the sum-rule values that have been derived from the multiplet simulations. The value of $\left\langle S E_{z}\right\rangle$ is then given as $\left\langle S_{z}\right\rangle$ $+7 / 2\left\langle T_{z}\right\rangle$.

The calculated value for $\left\langle L_{z}\right\rangle$ is found to be always exactly equal to the derived sum-rule value. This confirms the validity of the $\left\langle L_{z}\right\rangle$ sum rule. Because this sum rule integrates the complete $L$ edge, the internal structure of the $L$ edge due to spin-orbit coupling and multiplet effects has no effect on the integrated value. Except for Sec. III E, all other simulations were done at $0 \mathrm{~K}$.

\section{A. Case of the $\mathrm{Cu}^{2+} 3 d^{9}$ ground state}

The $3 d^{9}$ ground state has only a single $3 d$ hole. This implies that there are no $3 d 3 d$ two-electron integrals. The final state of the $2 p$ x-ray absorption process has a $2 p^{5} 3 d^{10}$ configuration, in other words a single $2 p$ hole, which implies that there are also no $2 p 3 d$ two-electron multiplet effects. The result is that there are no theoretical errors in applying the effective spin sum rule to $3 d^{9}$ systems. On the other hand, $\left\langle T_{z}\right\rangle$ is significant and some general aspects are discussed below. For an analytical deduction of the $\mathrm{Cu}^{2+}$ case as well as temperature dependence calculations, the reader is referred to Ref. 16.

If the $3 d$ spin-orbit coupling is zero, the $C u^{2+} 3 d^{9} \mathrm{~L}$ edge spectrum is characterized with a $L_{3}: L_{2}$ intensity ratio of $2: 1$ and a XMCD ratio of $-1: 1$. The $\left\langle S_{z}\right\rangle$ expectation value is -0.5 . Without $3 d$ spin-orbit coupling $\left\langle T_{z}\right\rangle$ is zero, implying that $\left\langle S E_{z}\right\rangle$ is also -0.5 . This is also exactly the value that is found after applying the sum rule to the $L$ edge spectrum.

If the $3 d$ spin-orbit coupling is not zero, the spin sum rule remains exact, but $\left\langle T_{z}\right\rangle$ will obtain a nonzero value. It turns out that for a $3 d^{9}$ ground state, the value of $\left\langle T_{z}\right\rangle$ is large. In atomic symmetry, using a cubic crystal field $(10 D q)$ of $0 \mathrm{eV}$, an atomic $3 d$ spin-orbit coupling of $\sim 0.1 \mathrm{eV}$, and an exchange field of $0.01 \mathrm{eV}$, one finds that all intensity is found in the transition $\mu_{-}\left(L_{3}\right)$. All transitions to the $L_{2}$ edge are zero and all transitions of $\mu_{+}$are zero. In other words $\mu_{+1}\left(L_{3}\right)$ $=\mu_{-1}\left(L_{2}\right)=\mu_{+1}\left(L_{2}\right)=0$. This implies that $\mu_{=} \mu_{-1}\left(L_{3}\right)$ and using Eq. (4),

$$
\left\langle S E_{z}\right\rangle=\frac{-\mu}{\mu} \frac{3}{2}\left\langle N_{h}\right\rangle=-1.5 .
$$

The value of $\left\langle S_{z}\right\rangle$ is -0.5 , which implies that the value of $7 / 2\left\langle T_{z}\right\rangle$ must be -1.0 . One can conclude that the effective spin value of $\left\langle S E_{z}\right\rangle=-1.5$ is exactly reproduced by the effective spin sum rule, but due to the large value of $\left\langle T_{z}\right\rangle$ this value of -1.5 is very far from the spin expectation value $\left\langle S_{z}\right\rangle$ of -0.5 .

Applying a cubic crystal field of $1.0 \mathrm{eV}$ yields a value for $\left\langle S E_{z}\right\rangle$ of -1.37 . The precise value is determined by a combination of the $3 d$ spin-orbit splitting, the exchange interaction, and the cubic crystal field and in fact for this Jahn-Teller system also by the effects of symmetry distortion to tetragonal symmetry. However, in all cases the effective spin sum rule remains correct. The fact that $7 / 2\left\langle T_{z}\right\rangle$ is equal to -1 implies that the hole is in a $3 d_{x^{2}-y^{2}}$ orbital. Without spin-orbit coupling this state is degenerate with a hole in a $3 d_{z^{2}}$ orbital, but the spin-orbit splitting causes a single $3 d$ hole in the $3 d_{x^{2}-y^{2}}$ orbital at $0 \mathrm{~K}$. At finite temperatures, the occupation is equivalent between $3 d_{x^{2}-y^{2}}$ and $3 d_{z^{2}}$ holes as the energy splitting is only $10^{-5} \mathrm{eV}$. In actual systems, the $3 d^{9}$ ground state is split by a Jahn-Teller distortion, usually an elongation of the $z$ axis toward a square planar symmetry. This again creates a single hole in the $3 d_{x^{2}-y^{2}}$ orbital and a $\left\langle T_{z}\right\rangle$ value of -1 . Without spin-orbit coupling the value of $7 / 2\left\langle T_{z}\right\rangle$ is (half) integer for all $3 d$ orbitals. It is +1 for the $3 d_{x^{2}-y^{2}}$ and $3 d_{x y}$ orbitals, it is -1 for the $3 d_{z^{2}}$ orbital, and it is $-1 / 2$ for the $3 d_{x z}$ and $3 d_{y z}$ orbitals. If the ground state of a material has its $3 d$ states split by a value larger than the $3 d$ spin-orbit coupling, one can directly derive the approximate values of $\left\langle T_{z}\right\rangle$ for all high-spin systems from $3 d^{1}$ to $3 d^{9}$. A $3 d^{1}$ configuration with an elongated $z$ axis has its $3 d_{x y}$ state occupied, a $3 d^{1}$ configuration with a compressed $z$ axis has its $3 d_{x z}$ and $3 d_{y z}$ states half occupied, etc.

\section{B. Effects of the crystal field splitting and the $3 d$ spin-orbit coupling}

We start by calculating the expectation values for systems between four and eight $3 d$ electrons, i.e., $\mathrm{Mn}^{3+} 3 d^{4}, \mathrm{Fe}^{3+} 3 d^{5}$, $\mathrm{Fe}^{2+} 3 d^{6}, \mathrm{Co}^{2+} 3 d^{7}$, and $\mathrm{Ni}^{2+} 3 d^{8}$, using atomic $2 p 3 d$ and $3 d 3 d$ Slater integrals, atomic $2 p$ spin-orbit coupling, and an internal exchange field of $10 \mathrm{meV}$. The $3 d$ spin-orbit coupling was varied between the atomic value and zero. The octahedral crystal field $(10 D q)$ is changed between 0.0 and $3.0 \mathrm{eV}$.

Figure 1 gives the expectation values of the spin $\left\langle S_{z}\right\rangle$, the spin-quadrupole contribution to the sum rule $7 / 2\left\langle T_{z}\right\rangle$, and the theoretical value of the effective spin $\left\langle S E_{z}\right\rangle$ as a function of $10 D q$. Different curves indicate calculations with distinct magnitudes for the $3 d$ spin-orbit coupling. The magnitude of $7 / 2\left\langle T_{z}\right\rangle$ plays an important role in the application of the sum 

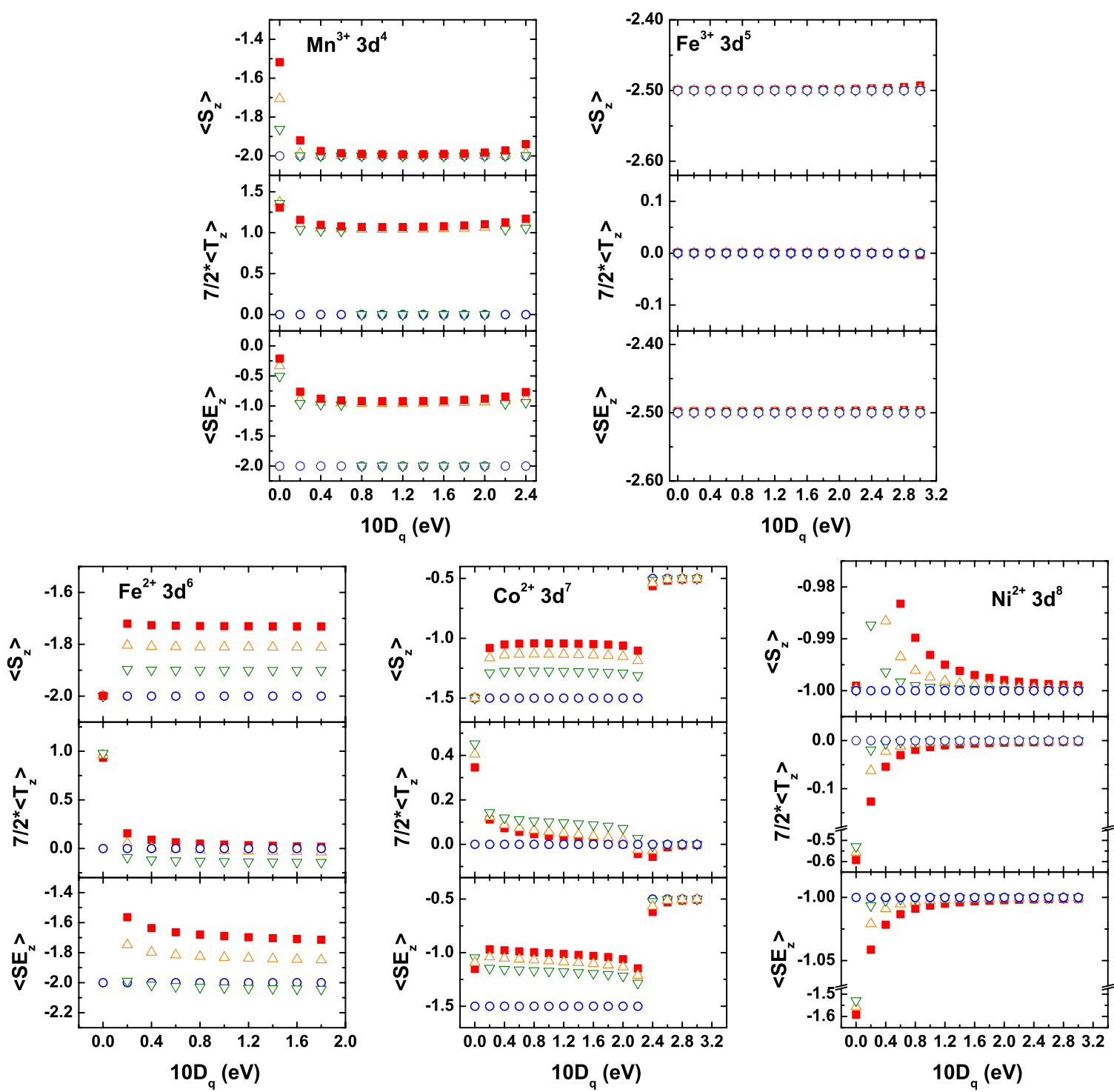

FIG. 1. (Color online) The expectation values of $\left\langle S_{z}\right\rangle, 7 / 2\left\langle T_{z}\right\rangle$, and $\left\langle S E_{z}\right\rangle$ are given as a function of the cubic crystal field splitting of $10 D q$. Given are (top, left) $\mathrm{Mn}^{3+} 3 d^{4}$, (top, right) $\mathrm{Fe}^{3+} 3 d^{5}$, (bottom, left) $\mathrm{Fe}^{2+} 3 d^{6}$, (bottom, middle) $\mathrm{Co}^{2+} 3 d^{7}$, and (bottom, right) $\mathrm{Ni}^{2+} 3 d^{8}$. The symbols indicate calculations with atomic $3 d$ spin-orbit coupling (filled square, red), $60 \%$ of the atomic value (up triangle, orange), $30 \%$ of the atomic value (down triangle, green), and no $3 d$ spin-orbit coupling (open circle, blue).

rules in experimental spectra since its value is often unknown and in most cases assumed to be negligible. Analyzing Fig. 1 it is seen that in case the atomic $3 d$ spin-orbit coupling is zero (open circles), the value of $\left\langle T_{z}\right\rangle$ is zero and $\left\langle S_{z}\right\rangle$ is given by -0.5 times the number of holes. A zero value for $\left\langle T_{z}\right\rangle$ also implies that $\left\langle S E_{z}\right\rangle=\left\langle S_{z}\right\rangle$. For all cubic $3 d^{6}, 3 d^{7}$, and $3 d^{8}$ systems with a crystal field above $0.5 \mathrm{eV}$, the value of $7 / 2\left\langle T_{z}\right\rangle$ is between -0.1 and 0.1 . In case of $3 d^{6}$ systems the sign of $\left\langle T_{z}\right\rangle$ depends on the magnitude of the $3 d$ spinorbit coupling. The contribution of $\left\langle T_{z}\right\rangle$ is therefore small and $\left\langle S E_{z}\right\rangle$ is very close to $\left\langle S_{z}\right\rangle$. In case of $3 d^{5}$ systems, $\left\langle T_{z}\right\rangle$ is always zero because the shell is half-filled. The $3 d^{4}$ systems present a special case with respect to the values of $\left\langle T_{z}\right\rangle$. One can observe that there are essentially two options for $\left\langle T_{z}\right\rangle,(1)$ a value close to zero or (2) a value close to 1.0. The origin for the value of 1.0 can be found in Table I. The $3 d$ spin-orbit coupling creates a small energy difference between the
$3 d_{x^{2}-y^{2}}$ and $3 d_{z^{2}}$ states. If only the $3 d_{z^{2}}$ state is occupied, the value of $\left\langle T_{z}\right\rangle$ is +1 . A value of 0.0 is found without $3 d$ spinorbit coupling and for a small spin-orbit coupling. In real systems, there will often be a distortion in the $3 d^{4}$ ground state implying a $\left\langle T_{z}\right\rangle$ value of -1 or +1 . One can use Fig. 1 to have an indication of the differences for the values of $\left\langle T_{z}\right\rangle$ being equal to 0 or +1 .

There is little change in the value of $\left\langle S_{z}\right\rangle$ as a function of the crystal field except for the $3 d^{7}$ diagrams, where a $S$ $=1.5$ high-spin to $S=0.5$ low-spin transition is visible at 2.3 $\mathrm{eV}$. The variation of $\left\langle S_{z}\right\rangle$ with the spin-orbit coupling magnitude is due to the competition between the spin-orbit coupling and the exchange energy. For the atomic spin-orbit coupling the exchange energy of $10 \mathrm{meV}$ is not enough to completely saturate the system for $\mathrm{Fe}^{2+}$ and $\mathrm{Co}^{2+}$. The effect of the exchange energy is studied in more detail in Sec. III D. $\left\langle S E_{z}\right\rangle$ is for all case equal to $\left\langle S_{z}\right\rangle+7 / 2\left\langle T_{z}\right\rangle$. In case of the $3 d^{4}$ 
TABLE I. The expectation values for $7 / 2\left\langle T_{z}\right\rangle$ in $D_{4 h}$ (square planar) symmetry for the different occupations of the $3 d$ states in case that the $3 d$ spin-orbit coupling and the $3 d 3 d$ interactions are set to zero. Both cases for an elongated and compressed octahedron are shown.

\begin{tabular}{ccc}
\hline \hline Conf. & $7 / 2\left\langle T_{z}\right\rangle$ (elongated) & $7 / 2\left\langle T_{z}\right\rangle$ (compressed) \\
\hline $3 d^{1}$ & $\frac{1}{2}$ & -1 \\
$3 d^{2}$ & 1 & $-\frac{1}{2}$ \\
$3 d^{3}$ & 0 & 0 \\
$3 d^{4}$ & 1 & -1 \\
$3 d^{5}$ & 0 & 0 \\
$3 d^{6}$ & $-\frac{1}{2}$ & 1 \\
$3 d^{7}$ & -1 & $\frac{1}{2}$ \\
$3 d^{8}$ & 0 & 0 \\
$3 d^{9}$ & -1 & 1 \\
\hline \hline
\end{tabular}

systems this yields again the two options: (1) $\left\langle S E_{z}\right\rangle=\left\langle S_{z}\right\rangle$ or (2) $\left\langle S E_{z}\right\rangle=\left\langle S_{z}\right\rangle+1.0$.

Figure 2 gives the ratio $\left[S E_{z}^{\mathrm{sum}}\right] /\left\langle S E_{z}\right\rangle$ (top panels) and $\left[S E_{z}^{\text {sum }}\right] /\left\langle S_{z}\right\rangle$ (bottom panels). A value of 1.0 implies that the sum-rule value $\left[S E_{z}^{\text {sum }}\right]$ is equal to the expectation values for $\left\langle S E_{z}\right\rangle$ and $\left\langle S_{z}\right\rangle$, respectively. The error in the spin sum rule is given by $\left[S E_{z}^{\text {sum }}\right] /\left\langle S E_{z}\right\rangle$. The ratio $\left[S E_{z}^{\text {sum }}\right] /\left\langle S_{z}\right\rangle$ is also given as the experimental quantity that one usually attempts to determine is $\left\langle S_{z}\right\rangle$. In case of $\mathrm{Ni}^{2+} 3 d^{8}$, the values for $\left[S E_{z}^{\text {sum }}\right] /\left\langle S E_{z}\right\rangle$ and $\left[S E_{z}^{\text {sum }}\right] /\left\langle S_{z}\right\rangle$ are close to 0.90 , except for the atomic calculations and calculations with very small crystal fields. This implies that for $3 d^{8}$ systems one finds an underestimation in $\left\langle S_{z}\right\rangle$ of approximately $10 \%$, independent of the precise value of the crystal field and also independent of the $3 d$ spin-orbit coupling. Figure 2 shows that for the $3 d^{7}$ systems $\left(\mathrm{Co}^{2+}\right)$, the $\left[S E_{z}^{\text {sum }}\right] /\left\langle S E_{z}\right\rangle$ value is approximately 0.92 with $3 d$ spin-orbit coupling and $\sim 0.84$ without $3 d$ spinorbit coupling. The value of $\left[S E_{z}^{\mathrm{sum}}\right] /\left\langle S_{z}\right\rangle$ lies between 0.84
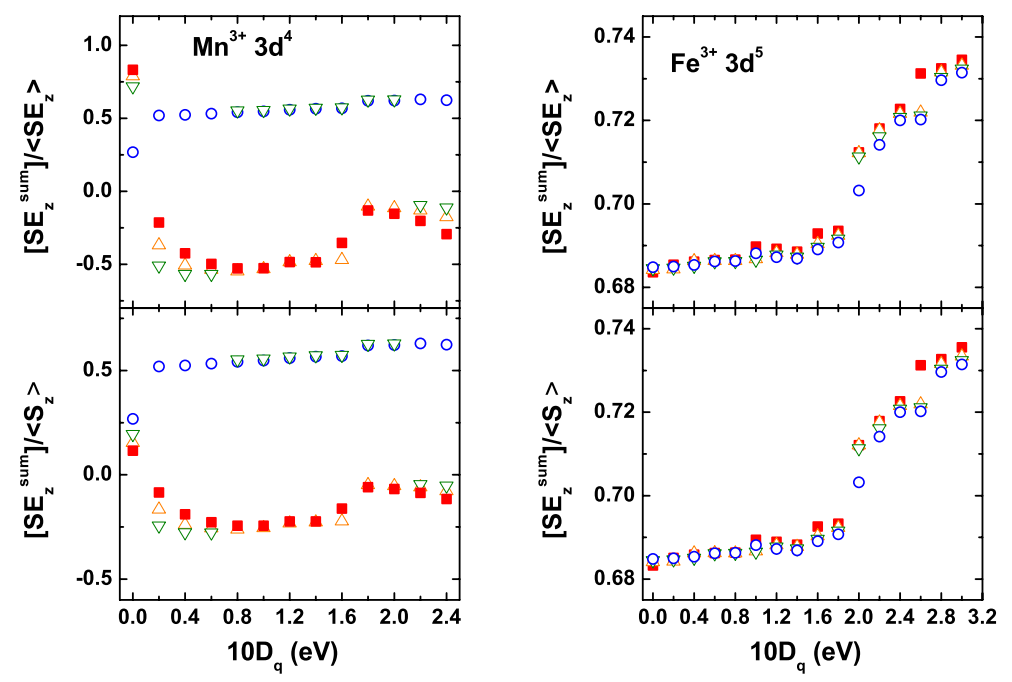
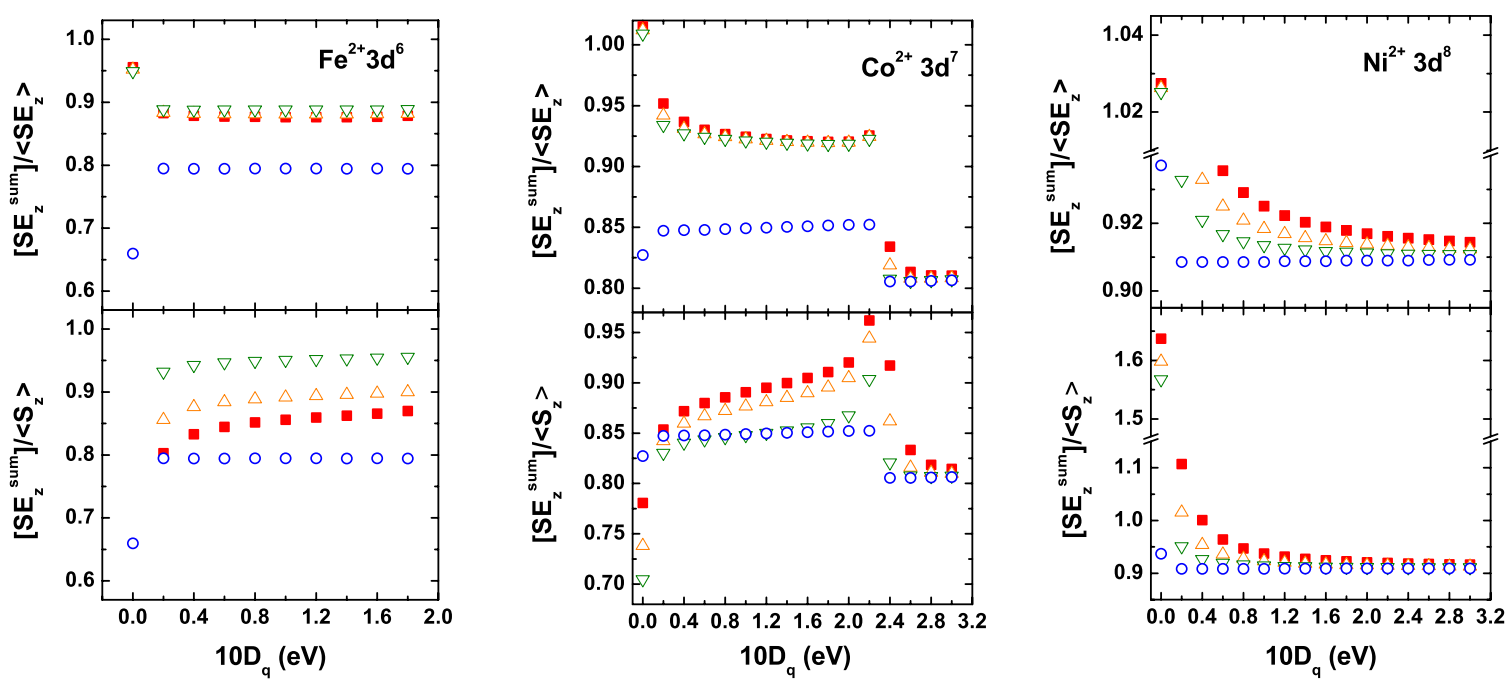

FIG. 2. (Color online) The ratio of the sum-rule value $\left[S E_{z}^{\text {sum }}\right]$ with $\left\langle S E_{z}\right\rangle$ (top panels) and $\left\langle S_{z}\right\rangle$ (bottom panels) for (top, left) $\mathrm{Mn}^{3+} 3 d^{4}$, (top, right) $\mathrm{Fe}^{3+} 3 d^{5}$, (bottom, left) $\mathrm{Fe}^{2+} 3 d^{6}$, (bottom, middle) $\mathrm{Co}^{2+} 3 d^{7}$, and (bottom, right) $\mathrm{Ni}^{2+} 3 d^{8}$. The symbols indicate calculations with atomic $3 d$ spin-orbit coupling (filled square, red), $60 \%$ of the atomic value (up triangle, orange), 30\% of the atomic value (down triangle, green), and no $3 d$ spin-orbit coupling (open circle, blue). 
and 0.96. At $10 D q=2.4$ a transition to a low-spin ground state is visible. All low-spin $3 d^{7}$ systems have an error value of $\sim 0.8$, implying a sum-rule error of $\sim 20 \%$, independent of crystal field strength and spin-orbit coupling.

The case of $\mathrm{Fe}^{2+} 3 d^{6}$ is similar to the $3 d^{7}$ ground state: a $\left[S E_{z}^{\text {sum }}\right] /\left\langle S E_{z}\right\rangle$ value of 0.8 without spin-orbit coupling and a value at $\sim 0.88$ with spin-orbit coupling. In case of $\left[S E_{z}^{\text {sum }}\right] /\left\langle S_{z}\right\rangle$, the values vary between 0.80 and 0.96 . This implies a $\left\langle S_{z}\right\rangle$ between $4 \%$ and $20 \%$, dependent on the values of $10 D q$ and the $3 d$ spin-orbit coupling. The $\mathrm{Fe}^{3+} 3 d^{5}$ systems have identical curves for $\left[S E_{z}^{\text {sum }}\right] /\left\langle S E_{z}\right\rangle$ and $\left[S E_{z}^{\text {sum }}\right] /\left\langle S_{z}\right\rangle$ as all values of $\left\langle T_{z}\right\rangle$ are zero. We observe values for $\left[S E_{z}^{\text {sum }}\right] /\left\langle S E_{z}\right\rangle$ and $\left[S E_{z}^{\text {sum }}\right] /\left\langle S_{z}\right\rangle$ between 0.68 and 0.74 , where these values are determined by the value of the cubic crystal field. This implies a systematic (uniform) underestimation of $\left\langle S_{z}\right\rangle$ by $\sim 30 \%$.

In case of $\mathrm{Mn}^{3+} 3 d^{4}$, there is little relation between the sum-rule value and the $\left\langle S E_{z}\right\rangle$ and $\left\langle S_{z}\right\rangle$ expectation values. In systems where $7 / 2\left\langle T_{z}\right\rangle=0$, in other words in systems where the two lowest states are degenerate, the values of $\left[S E_{z}^{\text {sum }}\right] /\left\langle S E_{z}\right\rangle$ and $\left[S E_{z}^{\text {sum }}\right] /\left\langle S_{z}\right\rangle$ are approximately 0.5 , implying an underestimation of $50 \%$ by the sum rule. If the $3 d$ spin-orbit coupling or alternatively a symmetry distortion splits these two lowest states, the $\left[S E_{z}^{\text {sum }}\right] /\left\langle S E_{z}\right\rangle$ value is between -0.2 and -0.5 and the value of $\left[S E_{z}^{\text {sum }}\right] /\left\langle S_{z}\right\rangle$ lies between 0.0 and -0.3 . This implies that the sum rule gives next to an underestimation of $50-80 \%$, also the wrong sign for the $\left\langle S E_{z}\right\rangle$ (and $\left.\left\langle S_{z}\right\rangle\right)$ value. For actual $3 d^{4}$ systems, it is not $a$ priori known if the ground state is degenerate or split; one does not know if the error of the effective spin sum rule is $50 \%$ or $-50 \%$, so one is not even sure of the sign of the (effective) spin from the derived sum-rule value.

It can be concluded that the case that $\left\langle T_{z}\right\rangle$ is close to zero does not imply that the spin sum rule is exact. As seen in Fig. 2 , the largest errors in the spin sum rule actually arise without $3 d$ spin-orbit coupling (for zero $\left\langle T_{z}\right\rangle$ values).

\section{Effect of multiplet interactions and the $2 p$ spin-orbit coupling}

Next we would like to determine which interactions play a role in the spin rule errors. First, we focus on the role of the final state interactions: the $2 p 3 d$ multiplet interactions and the $2 p$ spin-orbit coupling. These final state effects do not influence the ground state and as such do not modify the expectation values for $\left\langle S_{z}\right\rangle,\left\langle L_{z}\right\rangle$, and $\left\langle T_{z}\right\rangle$. They affect however the spectral shapes and as such they modify the values for $\left[S E_{z}^{\text {sum }}\right.$.

Figure 3 shows that changing the $2 p 3 d$ multiplet interactions $F_{2 p 3 d}$ and $G_{2 p 3 d}$ in $\mathrm{Ni}^{2+}$ from zero to their atomic values decreases the sum-rule value from its calculated value of -1.0 to a value of approximately -0.90 . The atomic values of the Slater integrals yield a $10 \%$ error. There is no error for a calculation without $2 p 3 d$ multiplet effects and the relation between the $2 p 3 d$ multiplet effects and the $\left[S E_{z}^{\text {sum }}\right]$ value is approximately linear. An interesting observation is that the error is almost completely due to the $F_{2 p 3 d}^{2}$ Slater integral, in other words due to the dipole-dipole interactions between the $2 p$ and $3 d$ holes. The exchange terms $\left(G_{2 p 3 d}^{1}\right.$ and $\left.G_{2 p 3 d}^{3}\right)$

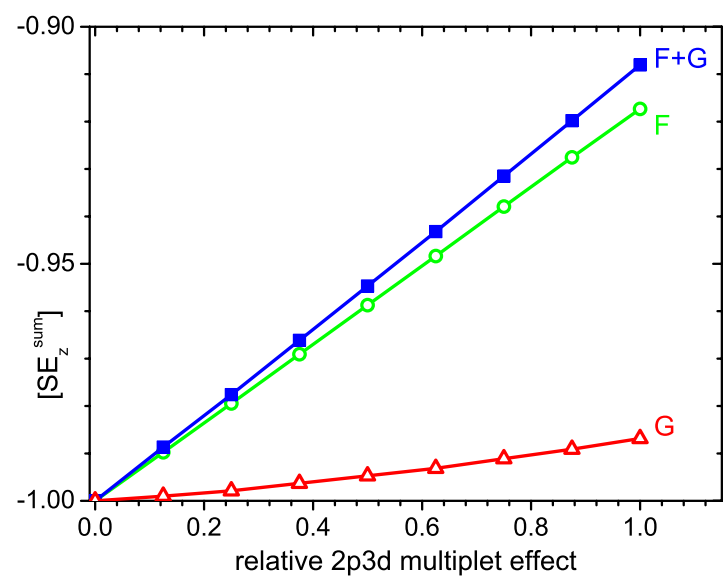

FIG. 3. (Color online) The sum-rule-derived value $\left[S E_{z}^{\mathrm{sum}}\right]$ expectation value for $\mathrm{Ni}^{2+}\left(d^{8}\right)$ as a function of the relative $2 p 3 d$ multiplet interactions $\left(F_{2 p 3 d}\right.$ and $\left.G_{2 p 3 d}\right)$, where 1.0 refers to the atomic Slater integral values. Three curves are given for only $G_{2 p 3 d}$ (open triangle, red), only $F_{2 p 3 d}$ (open circle, green), and for the combined effect of $G_{2 p 3 d}$ and $F_{2 p 3 d}$ (filled square, blue).

have little effect on the error, as is indicated by the triangles.

Figure 4 shows the spin expectation value as a function of the inverse $2 p$ spin-orbit coupling $\left(1 / \zeta^{\prime}\right)$, where $\zeta^{\prime}$ is normalized to the atomic value of the core hole spin-orbit coupling $\left(\zeta_{\text {atom }}\right)$ as $\zeta^{\prime}=\zeta / \zeta_{\text {atom }}$. The $\left[S E_{z}^{\text {sum }}\right] /\left\langle S E_{z}\right\rangle$ ratio at the atomic $2 p$ spin-orbit coupling is $\sim 10 \%$ in case of $\mathrm{Ni}^{2+}$. One observes that a larger $2 p$ spin-orbit coupling decreases the error. The error decreases linearly with $1 / \zeta^{\prime}$, implying that if the $2 p$ spin-orbit coupling is large, the effective spin sum rule is correct. Or, more specifically, if $\zeta_{2 p} /\left\langle F_{2 p 3 d}\right\rangle$ is large, the error in $\left\langle S E_{z}\right\rangle$ can be neglected. This also implies that the $L$ edges of the $4 d, 5 d$, and $4 f$ elements will have errors in $\left\langle S E_{z}\right\rangle$ close to zero, at least due to the multiplet and spinorbit induced effects.

\section{Effect of exchange field}

The calculations shown in Secs. III A-III C have used an exchange field of $10 \mathrm{meV}$ to split the ground state. As a first

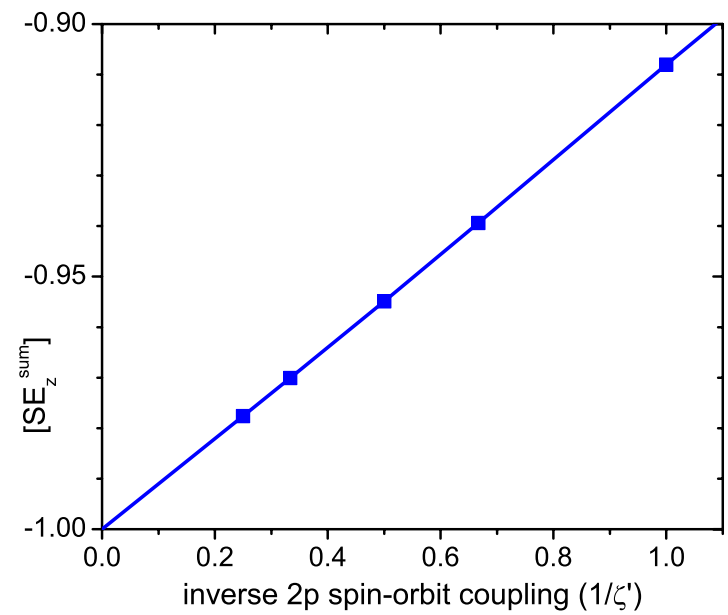

FIG. 4. (Color online) The $\left[S E_{z}^{\text {sum }}\right]$ value for $\mathrm{Ni}^{2+}\left(d^{8}\right)$ as a function of the inverse $2 p$ spin-orbit coupling $1 / \zeta^{\prime}$. 

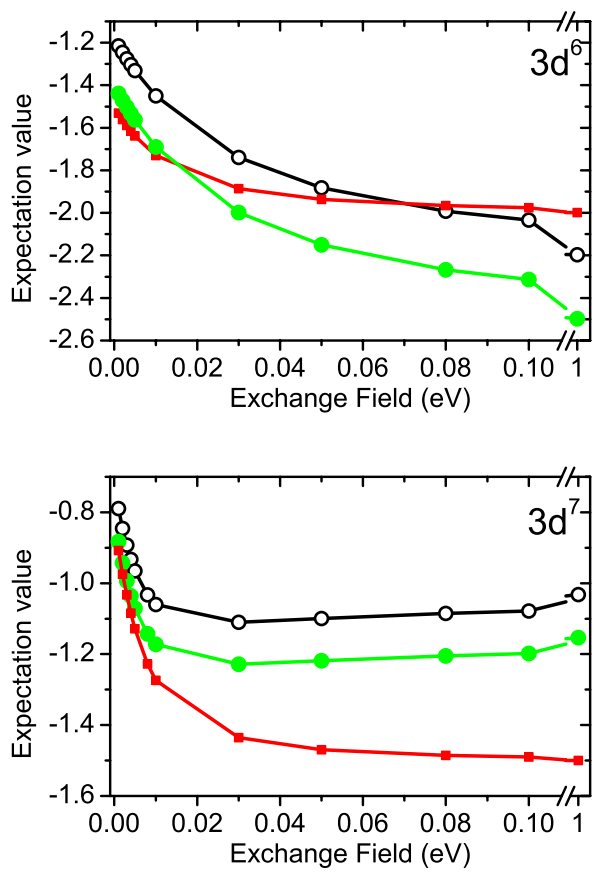

FIG. 5. (Color online) The expectation values $\left\langle S_{z}\right\rangle$ (red, small squares), $\left\langle S E_{z}\right\rangle$ (green, closed circles), and [SE $\left.E_{z}^{\text {sum }}\right]$ (black, open circles) as a function of the exchange field. Given are $\mathrm{Fe}^{2+} 3 d^{6}$ (top) and $\mathrm{Co}^{2+} 3 d^{7}$ (bottom) ground states.

approximation one can assume that the exchange field that should be used is given by the Curie temperature of the system. An exchange field of $10 \mathrm{meV}$ corresponds to a Curie temperature of approximately $116 \mathrm{~K}$. If the $3 d$ spin-orbit coupling is zero, the ground state can be indicated by a pure $L S$ term symbol. Such ground state will be evenly split by an exchange field. The magnitude of the exchange field determines the size of the splitting, but (for all practical exchange field values) it will not modify the nature of the states. This implies that (at $0 \mathrm{~K}$ ) the expectation values are independent of the exchange field.

Things change if the $3 d$ spin-orbit coupling is nonzero. The small but finite $3 d$ spin-orbit coupling splits the ground state into its double group states. These states are closely spaced and their nature is determined by a combination of all interactions: (i) the exchange interaction combined with (ii) the $3 d$ spin-orbit coupling, (iii) the $3 d 3 d$ interactions, (iv) the crystal field, and (v) translation symmetry (or band) effects.

Figure 5 shows the effect of the magnitude of the applied exchange field on the expectation values of the spin $\left\langle S_{z}\right\rangle$, the effective spin $\left\langle S E_{z}\right\rangle$, and the sum-rule-derived value $\left[S E_{z}^{\mathrm{sum}}\right]$. The values are given from 0 to $100 \mathrm{meV}$, where the $100 \mathrm{meV}$ values are similar to the saturated values (where we used a value of $1.0 \mathrm{eV})$. The difference between $\left\langle S_{z}\right\rangle$ and $\left\langle S E_{z}\right\rangle$ is again caused by the value of $7 / 2\left\langle T_{z}\right\rangle$. One can observe that the difference between $\left\langle S_{z}\right\rangle$ and $\left\langle S E_{z}\right\rangle$ slowly increases for $3 d^{6}$ and $3 d^{7}$. In case of a $3 d^{6}$ ground state $\left\langle T_{z}\right\rangle$ changes sign at an exchange field of approximately $10 \mathrm{meV}$.

One observes that for $3 d^{6}$, the $\left\langle S_{z}\right\rangle$ expectation values decreases from -1.5 to -2.0 with an increasing exchange field, where the value of -2.0 represents the fully spin-polarized case of four electrons. Similarly for $3 d^{7}$ the $\left\langle S_{z}\right\rangle$ expectation values decrease from -0.9 to -1.5 . Without exchange field the $3 d$ spin-orbit coupling mixes the spin-polarized state with other states, an effect that is counteracted by the exchange field. An exchange field of $100 \mathrm{meV}$ yields effectively a completely spin-polarized state. The effect on the value of $\left\langle S E_{z}\right\rangle$ is, in addition, determined by $\left\langle T_{z}\right\rangle$. In case of a $3 d^{6}$ ground state, we observe a decrease from -0.9 to -1.25 at a field of $30 \mathrm{meV}$, followed by a slight increase to -1.15 . This increase is due to the effect of $\left\langle T_{z}\right\rangle$. The $3 d^{7}$ ground state decreases from -1.4 to -2.5 . The difference between the $\left\langle S E_{z}\right\rangle$ expectation value and the sum-rule value remains approximately constant for all exchange fields. In other words, the exchange field has a significant effect on the $\left\langle S_{z}\right\rangle$ and $\left\langle T_{z}\right\rangle$ expectation values, but the sum-rule error remains essentially constant.

In case of the $3 d^{8}$ ground state of $\mathrm{Ni}^{2+}$, the exchange splitting has no effect on the expectation values. The reason is that the ground state has a ${ }^{3} A_{2}$ ground state, i.e., a filled $t_{2 g}$ band plus a half-filled $e_{g}$ band. A ${ }^{3} A_{2}$ state is a single $\left(T_{2}\right)$ state in double group symmetry and it is not affected by the $3 d$ spin-orbit coupling.

\section{E. Temperature dependence}

In this section we will present how a finite temperature changes the results obtained in Sec. III B. Figure 6 shows the expectation values $\left\langle S_{z}\right\rangle,\left\langle S E_{z}\right\rangle$, and $7 / 2\left\langle T_{z}\right\rangle$ and the sum-rule correction factors $\left[S E_{z}^{\text {sum }}\right] /\left\langle S E_{z}\right\rangle$ and $\left[S E^{\text {sum }}\right] /\left\langle S_{z}\right\rangle$ as a function of temperature. From all systems presented in Sec. III B only $\mathrm{Mn}^{3+}\left(3 d^{4}\right), \mathrm{Fe}^{2+}\left(3 d^{6}\right)$, and $\mathrm{Co}^{2+}\left(3 d^{7}\right)$ show a significant temperature dependence and therefore these are the only systems discussed in this section. These are exactly the systems for which $\left\langle T_{z}\right\rangle$ has a significant contribution to $\left\langle S E_{z}\right\rangle$. The calculations presented in Fig. 6 were done for $10 D q$ $=1.0 \mathrm{eV}$, exchange energy of $100 \mathrm{meV}$, and atomic $3 d$ spinorbit coupling. The exchange energy was considerably increased compared to Sec. III B to assure magnetic saturation at $300 \mathrm{~K}$.

For all three systems presented in Fig. $6\left[S E_{z}^{\mathrm{sum}}\right] /\left\langle S_{z}\right\rangle$ has an important dependence with temperature, approaching $\left[S E_{z}^{\text {sum }}\right] /\left\langle S E_{z}\right\rangle$ as temperature increases. This is a direct consequence of the decrease of the $\left\langle T_{z}\right\rangle$ contribution to the effective spin as temperature increases. With increasing temperature the spin-orbit split values are more equally populated which leads to a quenching of $\left\langle T_{z}\right\rangle .{ }^{9}$ For $\mathrm{Mn}^{3+}\left(3 d^{4}\right),\left[S E_{z}^{\text {sum }}\right] /\left\langle S E_{z}\right\rangle$ also changes significantly with temperature. This comes from the fact that for $3 d^{4}$ there is no straightforward separation between $L_{3}$ and $L_{2}$ XMCD and the spectral shape is different for each $3 d$ spin-orbit split ground state. As temperature increases, the different $3 d$ spin-orbit split states are populated and therefore $\left[S E_{z}^{\mathrm{sum}}\right] /\left\langle S E_{z}\right\rangle$ changes reaching saturation when all spin-orbit split states are equally populated. The increase in temperature has an equivalent effect in $\left[S E_{z}^{\mathrm{sum}}\right] /\left\langle S E_{z}\right\rangle$ as the decrease of the $3 d$ spin-orbit coupling, presented in Fig. 2.

For $\mathrm{Fe}^{2+}\left(3 d^{6}\right)\left[S E_{z}^{\text {sum }}\right] /\left\langle S E_{z}\right\rangle$ varies only around $3 \%$ from $0 \mathrm{~K}$ to room temperature. $\left[S E_{z}^{\text {sum }}\right] /\left\langle S_{z}\right\rangle$ is higher than 1.0 for low temperature and approaches $\left[S E_{z}^{\text {sum }}\right] /\left\langle S E_{z}\right\rangle$ as $\left\langle T_{z}\right\rangle$ goes from negative values to zero. Notice that in Fig. 1 the values 

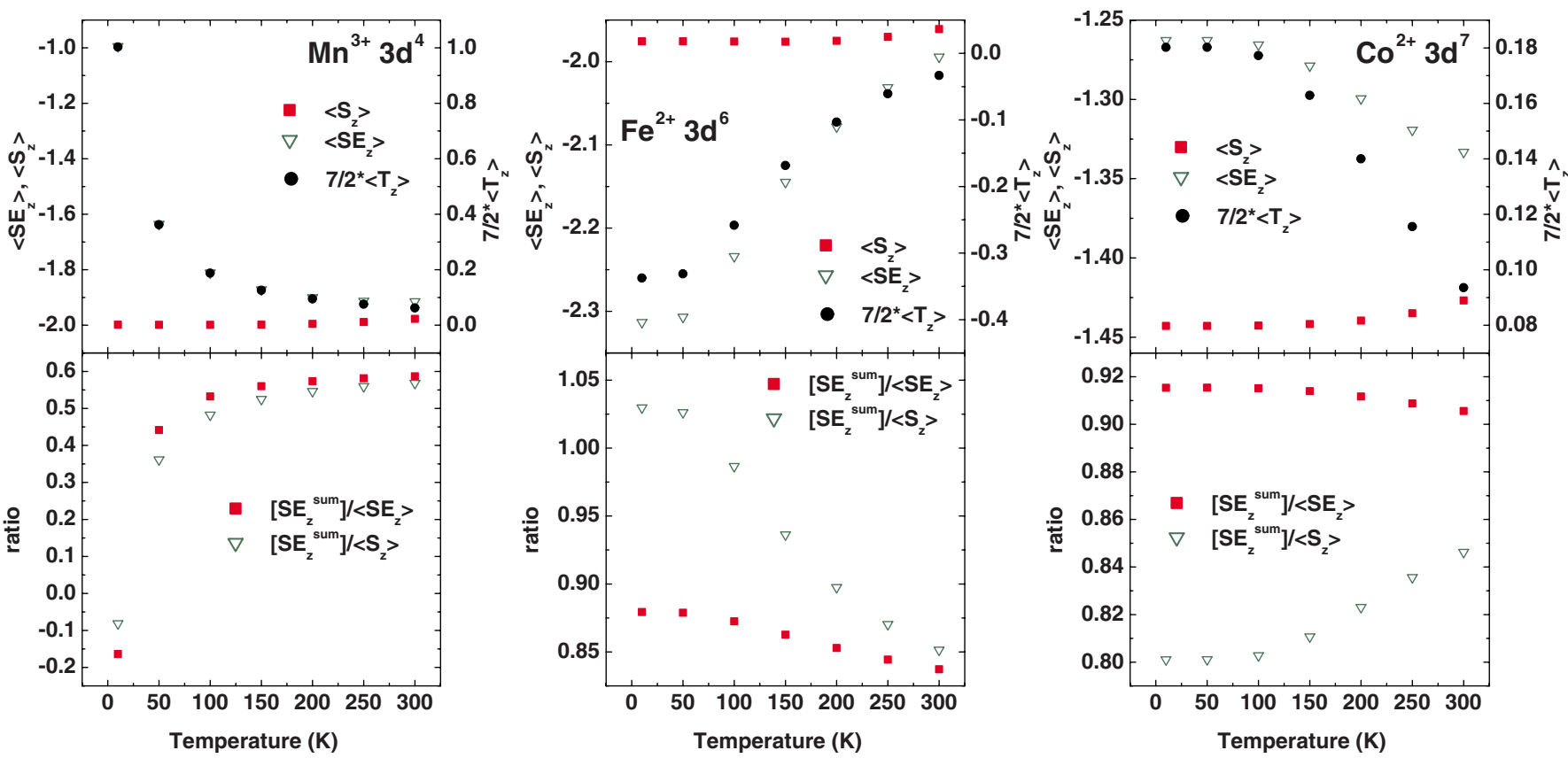

FIG. 6. (Color online) Temperature dependence for $\left\langle S_{z}\right\rangle,\left\langle S E_{z}\right\rangle, 7 / 2^{*}\left\langle T_{z}\right\rangle$, $\left[S E_{z}^{\text {sum }}\right] /\left\langle S E_{z}\right\rangle$, and $\left[S E_{z}^{\text {sum }}\right] /\left\langle S_{z}\right\rangle$ for $\mathrm{Mn}^{3+}\left(3 d^{4}\right), \mathrm{Fe}^{2+}\left(3 d^{6}\right)$, and $\mathrm{Co}^{2+}\left(3 d^{7}\right)$. Simulations done with $10 D q=1.0 \mathrm{eV}$ and exchange $=0.1 \mathrm{eV}$. The lowest temperature point is $10 \mathrm{~K}$.

of $\left\langle T_{z}\right\rangle$ are positive due to the different exchange energy used there (see in Fig. 5). $\left[S E_{z}^{\text {sum }}\right] /\left\langle S E_{z}\right\rangle$ for $\mathrm{Co}^{2+}\left(3 d^{7}\right)$ is almost constant with temperature varying only between 0.91 and 0.90 , while $\left[S E_{z}^{\text {sum }}\right] /\left\langle S_{z}\right\rangle$ varies between 0.80 and 0.85 .

\section{F. Effect of charge transfer}

Figure 7 gives the calculations of the $\left\langle S_{z}\right\rangle, 7 / 2\left\langle T_{z}\right\rangle$, and $\left\langle S E_{z}\right\rangle$ expectation values in the presence of charge transfer effects. We have applied charge transfer in a series of twostate calculations mixing $3 d^{N}$ with $3 d^{N+1} \underline{L}$. The parameters used were a spherical symmetric hopping $T$ of $2 \mathrm{eV}$ with varying charge transfer energy $\Delta$. Large positive $\Delta$ yields a pure $3 d^{N}$ ground state and large negative $\Delta$ yields a pure $3 d^{N+1} \underline{L}$ state. The expectation values are plotted as a function of the resulting $3 d$ occupancy for $\mathrm{Fe}^{2+}\left(3 d^{6}+3 d^{7} \underline{L}\right)$, $\mathrm{Co}^{2+}\left(3 d^{7}+3 d^{8} \underline{L}\right)$, and $\mathrm{Ni}^{2+}\left(3 d^{8}+3 d^{9} \underline{L}\right)$ calculations. Figure 7 shows calculation for two values of exchange fields: 10 and $500 \mathrm{meV}$.

With a large $500 \mathrm{meV}$ exchange field one observes for $\left\langle S_{z}\right\rangle$ in all cases a completely polarized ground state with only spin-down holes. The $3 d$ hole occupancy is for $100 \%$ spin-down holes, yielding a value of -0.5 times the number of holes $\left(\left\langle n_{h}\right\rangle\right)$, implying a straight line in the relationship between $\left\langle S_{z}\right\rangle$ and the $3 d$ occupancy. A smaller exchange field of $10 \mathrm{meV}$ is not able to counteract the effects of the $3 d$ spin-orbit coupling and incomplete polarization is visible in the value of $\left\langle S_{z}\right\rangle$. The values for $7 / 2\left\langle T_{z}\right\rangle$ are close to zero for a $3 d^{8}$ ground state. The values for $7 / 2\left\langle T_{z}\right\rangle$ are larger for the $500 \mathrm{meV}$ exchange field, where a $3 d^{7}$ state has a positive value of $\left\langle T_{z}\right\rangle$ and a $3 d^{6}$ ground state has a negative value. The resulting effective spin $\left\langle S E_{z}\right\rangle=\left\langle S_{z}\right\rangle+7 / 2\left\langle T_{z}\right\rangle$ deviates from a straight line as systems between $3 d^{7}$ and $3 d^{8}$ have higher values and systems between $3 d^{6}$ and $3 d^{7}$ have more negative values, both due to the effect of $\left\langle T_{z}\right\rangle$.

The resulting sum-rule errors of charge transfer calculations are given in Fig. 8. The $\left[S E_{z}^{\text {sum }}\right] /\left\langle S E_{z}\right\rangle$ ratio is between 0.88 and 0.95 in most cases, with values approaching 1.0 for

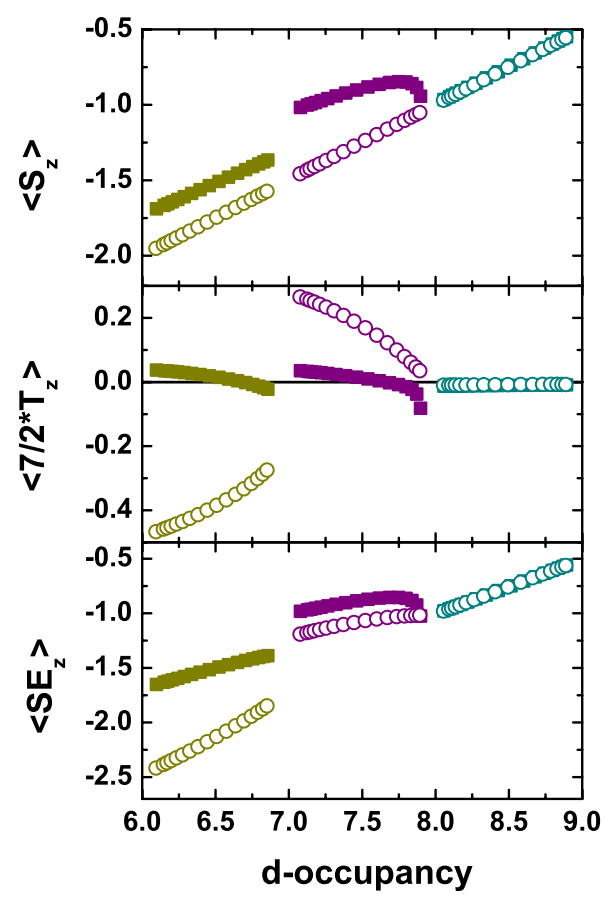

FIG. 7. (Color online) From top to bottom the expectation values $\left\langle S_{z}\right\rangle, 7 / 2\left\langle T_{z}\right\rangle$, and $\left\langle S E_{z}\right\rangle$ as a function of the $3 d$ occupancy of the ground states using a two-state charge transfer calculation are given. The exchange fields used are $10 \mathrm{meV}$ (closed squares) and $500 \mathrm{meV}$ (open circles). Results for $\mathrm{Fe}^{2+} d^{6}, \mathrm{Co}^{2+} d^{7}$, and $\mathrm{Ni}^{2+} d^{8}$ are showed in green, blue, and red, respectively. 


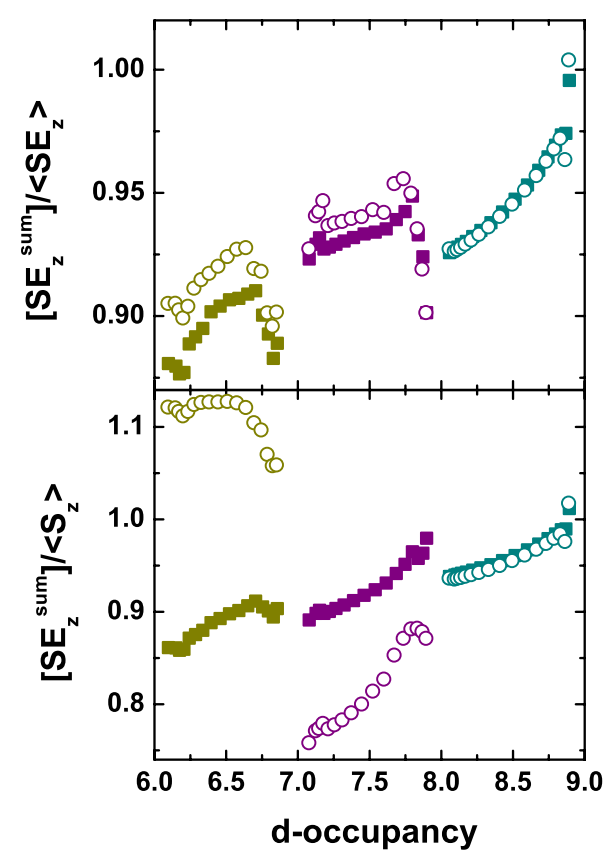

FIG. 8. (Color online) The ratio of the sum-rule value [SE $\left.E^{\text {sum }}\right]$ with $\left\langle S E_{z}\right\rangle$ (top) and $\left\langle S_{z}\right\rangle$ (bottom) as a function of the $3 d$ occupancy of the ground states using a two-state charge transfer calculation. The exchange fields used are $10 \mathrm{meV}$ (closed squares) and $500 \mathrm{meV}$ (open circles).

systems close to a $3 d^{9}$ ground state. These errors are relatively small. The $\left[S E_{z}^{\text {sum }}\right] /\left\langle S E_{z}\right\rangle$ ratio has been calculated taking the correct number of holes into account. The number of holes $\left\langle n_{h}\right\rangle$ is directly given as ten minus the number of $3 d$ electrons, which obviously varies with the charge transfer strength and considerably influences the value of $\left[S E_{z}^{\text {sum }}\right]$. The error in the spin expectation value, i.e., the $\left[S E_{z}^{\text {sum }}\right] /\left\langle S_{z}\right\rangle$ ratio, shows similar behavior for the $10 \mathrm{meV}$ exchange spectra since in those cases the value of $7 / 2\left\langle T_{z}\right\rangle$ is small. The curves for an exchange field of $500 \mathrm{meV}$ have errors that are dominated by $7 / 2\left\langle T_{z}\right\rangle$, with too small values for $\mathrm{Co}^{2+}$ and too large values for $\mathrm{Fe}^{2+}$.

\section{G. Effective spin sum rule for Ni metal}

The X-ray absorption and XMCD spectra of Ni metal have been simulated with the parameters from van der Laan and Thole. ${ }^{17}$ The calculation assumes three configurations in the ground state: $3 d^{8}+3 d^{9} \underline{L}+3 d^{10} \underline{L}^{2}$ at relative energies 0 , -2.25 , and $-3.0 \mathrm{eV}$. Hoppings of 0.7 and $1.4 \mathrm{eV}$ for $e_{g}$ and $t_{2 g}$ states were used. Crystal field splitting $(10 D q)$ is zero and exchange energy is $0.5 \mathrm{eV}$. The values obtained for the relative contributions of $3 d^{8}+3 d^{9} \underline{L}+3 d^{10} \underline{L}^{2}$ are $15 \%, 49 \%$, and $36 \%$, respectively. The sum-rule values obtained are summarized in Table II.

TABLE II. The expectation values obtained for Ni metal.

\begin{tabular}{ccccc}
\hline \hline & $\left\langle S_{z}\right\rangle$ & $7 / 2\left\langle T_{z}\right\rangle$ & $\left\langle S E_{z}\right\rangle$ & {$\left[S E_{z}^{\text {sum }}\right] /\left\langle S E_{z}\right\rangle$} \\
\hline Ni metal & -0.394 & -0.003 & -0.37 & 0.97 \\
\hline \hline
\end{tabular}

From Table II it is seen the $\left[S E_{z}^{\text {sum }}\right] /\left\langle S E_{z}\right\rangle$ value is close to 1 , showing that the application of the sum rule in isotropic metallic systems such as bulk Ni metal works well. The contribution of $\left\langle T_{z}\right\rangle$ to $\left\langle S E_{z}\right\rangle$ is also negligible for the case of $\mathrm{Ni}$ metal. From Fig. 8, it is clear that for $\mathrm{Co}$ and for Fe metal one would expect a more significant contribution of $7 / 2\left\langle T_{z}\right\rangle$.

\section{DISCUSSION}

We have analyzed the various parameters that influence the validity of the effective spin sum rule. The effective spin sum rule is correct for $\mathrm{Cu}^{2+}$ because the final state has a filled $3 d^{10}$ shell and is not affected by the $2 p 3 d$ intra-atomic interactions. The value for $7 / 2\left\langle T_{z}\right\rangle$ is however -1.0 for all octahedral systems at low temperature and also for tetragonal distorted (elongated) systems. For all systems that deviate from $O_{h}$ symmetry, a large value for $7 / 2\left\langle T_{z}\right\rangle$ is found. For $3 d^{5}, 3 d^{6}, 3 d^{7}$, and $3 d^{8}$ systems it was shown that, while in the same ground state symmetry, the crystal field splitting has almost no effect on $\left[S E_{z}^{\mathrm{sum}}\right] /\left\langle S E_{z}\right\rangle$. On the other hand a difference between high-spin and low-spin states is always observed. The ratio $\left[S E_{z}^{\text {sum }}\right] /\left\langle S_{z}\right\rangle$ shows small dependences with crystal field due to the $\left\langle T_{z}\right\rangle$ contribution. The $3 d$ spinorbit coupling has some influence on the $\left[S E_{z}^{\mathrm{sum}}\right] /\left\langle S E_{z}\right\rangle$ ratio for $3 d^{6}$ and $3 d^{7}$ systems and this dependence is also reflected in the $\left[S E_{z}^{\text {sum }}\right] /\left\langle S_{z}\right\rangle$ values. For $\mathrm{Mn}^{3+} 3 d^{4}$ system $\left[S E_{z}^{\text {sum }}\right] /\left\langle S E_{z}\right\rangle$ varies not only in magnitude but also in sign with crystal field splitting showing that in this case an application of the sum rule is practically impossible. For $3 d^{5}$ systems the sum-rule errors are seemly large: 0.68 for high spin and 0.74 for low spin. However it has no dependence on crystal field splitting or $3 d$ spin-orbit coupling. For $3 d^{6}, 3 d^{7}$, and $3 d^{8}$ systems the correction factors range between 0.8 and 0.9 .

In Sec. III C, we found that the effective spin sum-rule error scales linearly with $F_{p d}^{2} / L S_{2 p}$, in agreement with previous determinations. A remarkable result is that it is not the $2 p 3 d$ exchange interaction that is the origin of the error but instead the dipole-dipole interaction between the $2 p$ hole and the $3 d$ hole.

In systems with charge transfer the ratio $\left[S E_{z}^{\text {sum }}\right] /\left\langle S E_{z}\right\rangle$ is around 0.9 and 1 , showing therefore small dependence on the charge transfer amount. However, in the calculations the number of holes is known, which in the experimental case would be the limiting factor for the sum-rule application in systems with charge transfer.

\section{A. Potential derivation of a general correction factor}

We analyze the calculated results here with regard to the question if one can derive a correction factor which would make it possible to derive the $\left\langle S E_{z}\right\rangle$ or even $\left\langle S_{z}\right\rangle$ from the derived sum-rule value $\left[S E_{z}^{\text {sum }}\right]$. As mentioned in Sec. I, Goering et $a l .{ }^{13}$ developed such renormalization technique to derive the spin moment from the effective spin sum rule. An important factor in such correction factor is the branching ratio $[B]$. Just as the effective spin sum rule, the branching ratio is affected by the $2 p 3 d$ multiplet effects. ${ }^{18-20}$ Analysis of the effect of the $2 p 3 d$ multiplet effect on the effective spin 


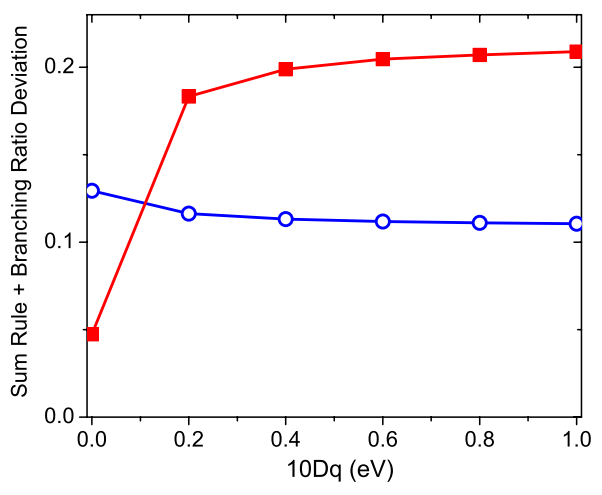

FIG. 9. (Color online) The variation of the deviation of the sum-rule value $\left[S E_{z}\right]^{\text {ERR }}$ (blue, open circles) and the value of the deviation from the statistical branching ratio $[\alpha]$ (red, closed squares) for $\mathrm{Fe}^{2+}\left(3 d^{6}\right)$.

sum rule yields an error that is linearly dependent on the magnitude of the multiplet effect, as was shown in Fig. 3. It can be shown that also the branching ratio is linearly dependent on the multiplet effect (see Fig. 6 in Ref. 19). This brings us to the following reasoning:

(1) The error in the spin sum rule is linearly dependent on the $2 p 3 d$ multiplet effects.

(2) The branching ratio is also linearly dependent on the $2 p 3 d$ multiplet effects.

(3) This implies that the deviation in the effective spin sum rule and the deviation in the branching ratio are correlated and one can calculate the effective spin deviation from the branching ratio deviation.

We define the transferred intensity between the $L_{2}$ and the $L_{3}$ edges as $[\alpha]$, where $[\alpha]$ is directly given by the branching ratio $[B]$ as $[\alpha]=[B]-2 / 3$. Note that $[B]$ and thus $[\alpha]$ can be derived from the experimental spectra without any theoretical input. We also define the error in the effective spin sum rule as $\left[S E_{z}\right]^{\mathrm{ERR}}=\left\langle S E_{z}\right\rangle-\left[S E_{z}\right]$ and we propose a linear relation between $\left[S E_{z}\right]^{\text {ERR }}$ and $[\alpha]$, in other words $\left[S E_{z}\right]^{\text {ERR }}$ $=f[\alpha]$. From the actual calculations for $\mathrm{Ni}^{2+}$ with a crystal field of $1.0 \mathrm{eV}$, we derive indeed a factor $f$ equal to -0.25 , with a deviation of $\sim 0.01$. This implies that for $\mathrm{Ni}^{2+}$ system the error in the effective spin sum rule can be corrected with a final accuracy of less than $0.5 \%$, where we note that this applies for the effective spin sum rule. This analysis and correction do not involve the value of $\left\langle T_{z}\right\rangle$.

So, have we now derived a useful correction procedure for the effective spin sum rule? Unfortunately not. The correlation between branching ratio and the effective spin sum rule is only valid as a function of the $2 p 3 d$ multiplet effects. If one varies the ground state, for example, a crystal field parameter, distortion, charge transfer effects, or the spin-orbit coupling, there is no linear relation between the $[\alpha]$ and $\left[S E_{z}\right]^{\mathrm{ERR}}$. For example, Fig. 9 shows the example of $[\alpha]$ and $\left[S E_{z}\right]^{\text {ERR }}$ in the case of $\mathrm{Fe}^{2+}$ as a function of $10 \mathrm{Dq}$. There is no simple relation and thus no general correction rule for the effective spin sum rule applies. The best procedure to correct the $\left[S E_{z}\right]$ values is to simulate the XAS and XMCD spectra and then to calculate the expectation values directly on the ground state. A general approach to determine an effective spin and spin correction procedure could be the following:
(1) Simulate the experimental spectrum with charge transfer multiplet calculations.

(2) Calculate the $\left\langle T_{z}\right\rangle$ and $\left\langle S_{z}\right\rangle$ expectation values for the as-such determined ground state.

(3) Calculate the theoretical sum-rule value $\left[S E_{z}^{\mathrm{sum}}\right]$ for this ground state.

(4) Determine the theoretical sum-rule errors $\left[S E_{z}^{\mathrm{sum}}\right] /\left\langle S E_{z}\right\rangle$ and $\left[S E_{z}^{\mathrm{sum}}\right] /\left\langle S_{z}\right\rangle$.

(5) Use this correction for the experimentally determined sum-rule value.

\section{B. Experimental effective spin sum-rule values}

In principle, the experimental sum-rule value and the theoretical sum-rule value should be the same, but the experiment is, in addition to the theoretical issues discussed here, affected by a number of additional aspects (see also Ref. 21), including the following:

E1. The number of holes in the accepting band plays a role because of the normalization to the overall XAS intensity. The number of holes is not always known experimentally.

E2. The $L_{3}$ and $L_{2}$ edges must be separable in order to determine the independent integrations, including the subtraction of the backgrounds. In addition, the appropriate edges must be separated from other structures and the continuum edge jump. In general this is a nontrivial task, with some variation in the methods used.

E3. If there is an angle between the x-ray beam and the magnetization vector, there is an x-ray absorption due to $\mu_{0}$, in addition to $\mu_{-}$and $\mu_{+}$. This effect can be neglected if the XAS spectrum of $\mu_{0}$ is given by the average of $\mu_{-}$and $\mu_{+}$, which would imply that the linear dichroism effect $\left(\mu_{-}+\mu_{+}\right.$ $\left.-2 \mu_{0}\right)$ is zero, an assumption that in general is not correct.

E4. If electron yield is used, the detection effectiveness must be equal for spin-up and spin-down electrons. This also implies that the escape chance for spin-up and spin-down electrons must be equal and in turn that the electron scattering should be spin independent.

E5. If fluorescence yield (FY) is used, there can be an angular and energy dependence of the signal distorting the XAS spectrum and also its associated XMCD signal. In addition, the FY signal is often affected by state (=energy) dependent variations in the measured signal. ${ }^{22}$

E6. When measuring by total electron yield (TEY), saturation effects will occur when the probing depth is of the order or smaller than the electron escaping depth. This is the case for very thin films or for measurements in grazing angles. Correction factors need to be applied to the x-ray absorption intensity. ${ }^{23,24}$

\section{Examples from experiments}

The experimentally determined sum-rule values are affected by two types of errors or inaccuracies: (1) due to the experimental procedures as described above and (2) due to the intrinsic theoretical errors for the (effective) spin sum rule. In order to verify the applicability of the individual orbital and spin sum rules, Chen et al. ${ }^{25}$ determined the values of $\left\langle L_{z}\right\rangle$ and $\left\langle S_{z}\right\rangle$, where both values were $\sim 5-10 \%$ too 
small for Fe and 5\% too large for Co. For the orbital moment, there is no theoretical error, but for $\left\langle S_{z}\right\rangle$ one would expect values that deviate between $5 \%$ and $20 \%$ (cf. Fig. 8). Apparently the experimental uncertainties dominate in this case. In Ref. 24 the values for $\left\langle S_{z}\right\rangle$ and $\left\langle L_{z}\right\rangle$ obtained by the sum rules are compared to neutron diffraction data for Fe, $\mathrm{Co}$, and $\mathrm{Ni}$ metal. The ratio $\left\langle S_{z}^{\mathrm{XMCD}}\right\rangle /\left\langle S_{z}^{\text {neutron }}\right\rangle$ (estimated from Fig. 8 in Ref. 24) is around 0.9, while we obtained 0.97 in Sec. III G. This is a satisfactory agreement in view of the experimental uncertainties involved in the determination of $\left\langle S_{z}^{\mathrm{XMCD}}\right\rangle$. In Ref. 26 it is found a correction factor of $1 / 1.47=0.68$ for $\mathrm{Mn}^{2+}\left(3 d^{5}\right)$. This correction factor is found with the help of theoretical simulations and it is the same as obtained here for $\mathrm{Fe}^{3+}\left(3 d^{5}\right)$.

Khanra et al. calculated the $L$ edge spectrum of a molecular $\mathrm{Mn}_{4} \mathrm{O}_{6}$ core system, with $\mathrm{Mn}^{2+}$ ions. ${ }^{27}$ They found a deviation in the spin sum-rule value of $\sim 30 \%$, exactly in agreement with Fig. 2 as given in this paper. This confirms the larger errors for $3 d^{5}$ systems. Gambardella et al. studied $\mathrm{Fe}, \mathrm{Co}$, and $\mathrm{Ni}$ atoms on a potassium surface. ${ }^{28}$ They found exactly correct $\left\langle S E_{z}\right\rangle$ values for the $3 d^{9}$ system $\mathrm{Ni}^{+}$, in agreement with theory as a $3 d^{9}$ system has no deviation for the effective spin sum rule. Actually for the atomic $3 d^{8}$ and $3 d^{7}$ systems, the theoretical error is also very small, $+3 \%$ for $3 d^{8}$ and $+1 \%$ for $3 d^{7}$, provided that the $3 d$ spin-orbit coupling is not quenched. The experimental data on $\mathrm{Co}^{+} 3 d^{8}$ are $\sim 10 \%$ too small and for $\mathrm{Fe}^{+} 3 d^{7}$ it is $\sim 20 \%$ too large, which are likely due to experimental aspects as discussed in the paper.

\section{CONCLUDING REMARKS}

We have analyzed the validity of the effective spin sum rule. In case of the $3 d^{9}$ ground state of $\mathrm{Cu}^{2+}$, the effective sum-rule value is exactly correct because the final state has a filled $3 d$ band and also there are no initial state or final state multiplet effects. The value of $7 / 2\left\langle T_{z}\right\rangle$ is large $(-1.0)$, implying that the effective spin is largely different from the spin moment $\left\langle S_{z}\right\rangle$.

The effective spin sum-rule errors for the $3 d^{4}-3 d^{9}$ systems as a function of (1) the crystal field effects and (2) the
$3 d$ spin-orbit coupling show errors of $5-10 \%$ for $\mathrm{Ni}^{2+} 3 d^{8}$ and $5-20 \%$ for $\mathrm{Co}^{2+} 3 d^{7}$ and also for $\mathrm{Fe}^{2+} 3 d^{6}$. The error for $\mathrm{Mn}^{2+} 3 d^{5}$ is approximately $30 \%$ and for the case of a $\mathrm{Mn}^{3+} 3 d^{4}$ ground state, the error is very large and varies between $-50 \%$ and $+50 \%$. This implies that, without further information, the derived effective spin sum-rule values for $\mathrm{Mn}^{3+} 3 d^{4}$ have essentially no meaning. The $3 d^{4}$ ground state is strongly affected by the Jahn-Teller distortion, which is strongly linked with the magnitude of the $\left\langle T_{z}\right\rangle$ value.

The simulations confirm that the final state effects of the $2 p 3 d$ multiplet effects and the core hole $2 p$ spin-orbit coupling are linearly related with the effective spin sum-rule error, that is, the error scales exactly with $\left\langle F_{2 p 3 d}\right\rangle / \zeta_{2 p}$, in agreement with previous results. Increasing the molecular exchange field saturates the spin moment and the value of $\left\langle T_{z}\right\rangle$ while maintaining the error in the sum-rule value for the whole range of applied fields.

The inclusion of charge transfer effects create a range of ground states with varying $3 d$ occupation, where we have in detail studied the occupation range between 6 and 9. For large exchange fields the spin moment is saturated, but $7 / 2\left\langle T_{z}\right\rangle$ is large, except for the range between 8 and 9. For small exchange fields, $7 / 2\left\langle T_{z}\right\rangle$ is small. The error in $\left\langle S E_{z}\right\rangle$ is between $5 \%$ and $10 \%$ for the whole parameter range, implying that for covalent and/or metallic systems the effective spin sum rule is precise to within 5-10\%. Because the sum rule always yields a too small value, a correction with $+5 \%$ will limit the error to less than $5 \%$. Because of the large $7 / 2\left\langle T_{z}\right\rangle$ values, the spin moment cannot reliably be determined from the effective spin sum rule with deviation between $-20 \%$ and $+10 \%$. It turns out to be not possible to derive a general correction method based on the branching ratio. Such correction is only possible for systems with similar ground states.

\section{ACKNOWLEDGMENT}

P.M. and F.M.F.d.G. acknowledge financial support from The Netherlands National Science Foundation (NWO/VICI program).
${ }^{1}$ B. T. Thole, P. Carra, F. Sette, and G. van der Laan, Phys. Rev. Lett. 68, 1943 (1992).

${ }^{2}$ P. Carra, B. T. Thole, M. Altarelli, and X. D. Wang, Phys. Rev. Lett. 70, 694 (1993).

${ }^{3}$ H. Wende, Rep. Prog. Phys. 67, 2105 (2004).

${ }^{4}$ T. Funk, A. Deb, S. J. George, H. X. Wang, and S. P. Cramer, Coord. Chem. Rev. 249, 3 (2005).

${ }^{5}$ F. M. F. de Groot, J. Electron Spectrosc. Relat. Phenom. 67, 529 (1994).

${ }^{6}$ J. Stohr and H. C. Siegmann, Magnetism: From Fundamentals to Nanoscale Dynamics (Springer-Verlag, Berlin, 2006).

${ }^{7}$ Y. Teramura, A. Tanaka, and T. Jo, J. Phys. Soc. Jpn. 65, 1053 (1996).

${ }^{8}$ G. van der Laan, K. T. Moore, J. G. Tobin, B. W. Chung, M. A. Wall, and A. J. Schwartz, Phys. Rev. Lett. 93, 097401 (2004).
${ }^{9}$ J. P. Crocombette, B. T. Thole, and F. Jollet, J. Phys.: Condens. Matter 8, 4095 (1996).

${ }^{10}$ G. van der Laan, C. M. B. Henderson, R. A. D. Pattrick, S. S. Dhesi, P. F. Schofield, E. Dudzik, and D. J. Vaughan, Phys. Rev. B 59, 4314 (1999).

${ }^{11}$ R. Q. Wu, D. S. Wang, and A. J. Freeman, Phys. Rev. Lett. 71, 3581 (1993).

${ }^{12}$ R. Q. Wu and A. J. Freeman, Phys. Rev. Lett. 73, 1994 (1994).

${ }^{13}$ E. Goering, S. Gold, and A. Bayer, Appl. Phys. A 78, 855 (2004).

${ }^{14}$ F. de Groot, Coord. Chem. Rev. 249, 31 (2005).

${ }^{15}$ F. de Groot and A. Kotani, Core Level Spectroscopy of Solids, 3rd ed. (Taylor \& Francis, New York, 2008).

${ }^{16}$ P. Sainctavit, M. A. Arrio, and C. Brouder, Phys. Rev. B 52, 12766 (1995). 
${ }^{17}$ G. van der Laan and B. T. Thole, J. Phys.: Condens. Matter 4, 4181 (1992).

${ }^{18}$ B. T. Thole and G. van der Laan, Phys. Rev. A 38, 1943 (1988).

${ }^{19}$ B. T. Thole and G. van der Laan, Phys. Rev. B 38, 3158 (1988).

${ }^{20}$ G. van der Laan and B. T. Thole, Phys. Rev. B 42, 6670 (1990).

${ }^{21}$ G. van der Laan, J. Synchrotron Radiat. 6, 694 (1999).

${ }^{22}$ F. M. F. de Groot, M. A. Arrio, Ph. Sainctavit, Ch. Cartier, and C. T. Chen, Solid State Commun. 92, 991 (1994).

${ }^{23}$ R. Nakajima, J. Stohr, and Y. U. Idzerda, Phys. Rev. B 59, 6421 (1999).

${ }^{24}$ W. L. O'Brien and B. P. Tonner, Phys. Rev. B 50, 12672 (1994).

${ }^{25}$ C. T. Chen, Y. U. Idzerda, H. J. Lin, N. V. Smith, G. Meigs, E.
Chaban, G. H. Ho, E. Pellegrin, and F. Sette, Phys. Rev. Lett. 75, 152 (1995).

${ }^{26}$ K. W. Edmonds, N. R. S. Farley, T. K. Johal, G. van der Laan, R. P. Campion, B. L. Gallagher, and C. T. Foxon, Phys. Rev. B 71, 064418 (2005).

${ }^{27}$ S. Khanra, K. Kuepper, T. Weyhermuller, M. Prinz, M. Raekers, S. Voget, A. V. Postnikov, F. M. F. de Groot, S. J. George, M. Coldea, M. Neumann, and P. Chaudhuri, Inorg. Chem. 47, 4605 (2008).

${ }^{28}$ P. Gambardella, S. S. Dhesi, S. Gardonio, C. Grazioli, P. Ohresser, and C. Carbone, Phys. Rev. Lett. 88, 047202 (2002). 